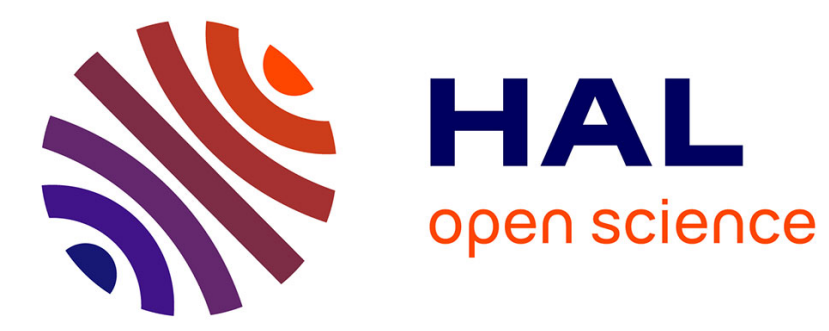

\title{
Multi-angle near infrared spectroscopy associated with common components and specific weights 5 analysis for in line monitoring
}

Maud Rey-Bayle, Ryad Bendoula, N. Caillol, Jean-Michel Roger

\section{- To cite this version:}

Maud Rey-Bayle, Ryad Bendoula, N. Caillol, Jean-Michel Roger. Multi-angle near infrared spectroscopy associated with common components and specific weights 5 analysis for in line monitoring. Journal of Near Infrared Spectroscopy, 2019, 27 (2), pp.134-146. 10.1177/0967033519830062 . hal02182372

\section{HAL Id: hal-02182372 \\ https://hal-ifp.archives-ouvertes.fr/hal-02182372}

Submitted on 12 Jul 2019

HAL is a multi-disciplinary open access archive for the deposit and dissemination of scientific research documents, whether they are published or not. The documents may come from teaching and research institutions in France or abroad, or from public or private research centers.
L'archive ouverte pluridisciplinaire HAL, est destinée au dépôt et à la diffusion de documents scientifiques de niveau recherche, publiés ou non, émanant des établissements d'enseignement et de recherche français ou étrangers, des laboratoires publics ou privés. 


\section{Corresponding Author:}

2 Maud Rey-Bayle, IFP Energies nouvelles, Rond-point de l'échangeur de Solaize, 69360 Solaize, France

3 Email: maud.rey-bayle@ifpen.fr

4 Multi-angle near infrared spectroscopy associated with common components and specific weights

$5 \quad$ analysis for in line monitoring

6

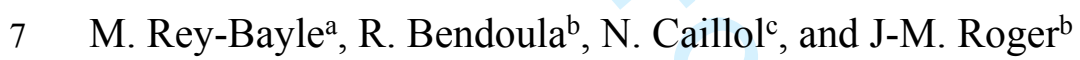

8 aIFP Energies nouvelles, Rond-point de l'échangeur de Solaize, BP3, 69360, Solaize, France

9 b ITAP, Irstea, Montpellier SupAgro, University of Montpellier, Montpellier, France

'AXEL’ONE, Rond-point de 1'échangeur de Solaize, 69360, Solaize, France

Abstract

Near infrared (NIR) spectroscopy offers a number of important advantages for process monitoring. In addition to its numerous practical advantages, an important reason to use NIR spectroscopy for process monitoring is its ability to supply versatile and multivariate information. However, in heterogeneous samples the interaction of light is complex and includes transmission, absorption and scattering simultaneously which all affect spectra. The measurement of the signal at one point may be insufficient. A solution is to measure the medium at several points and to use specific multivariate analysis.

In our study we propose to associate multipoint measurements with a Common Components and Specific Weight Analysis (CCSWA). We monitored two media online by angular multipoint Near infrared (NIR) 1 
spectroscopy. For the first medium, in which only the scattering varies over time, the precipitation of silica was chosen to illustrate such a medium. For the second medium, both scattering and absorption vary, whereby microemulsions implemented for enhanced oil recovery illustrate this medium. The results showed, by combining multi-angle measurements to CCSWA, the interest of measuring at different angles. In the first case, two scattering regimes have been identified and it was possible to access the anisotropy coefficient during the silica precipitation reaction. In the second case study, on microemulsions, it was possible to identify the different phases, and to separate the phenomena related to absorption and those related to diffusion.

These encouraging results validate the interest of coupling multi-angle measurements with multivariate multiblock analysis tools.

\section{Keywords}

Near infrared spectroscopy, multipoint, multi-angle, common components specific weights analysis, in line, monitoring, silica, EOR, microemulsions

\section{Introduction}

Process control is a topic of increasing research in recent years ${ }^{1}$ in the chemical industry and, is now even regulated for the pharmaceutical industry ${ }^{2}$. As mentioned by Kessler et al ${ }^{3}$ "cost, pressure, globalization and quality assurance will undoubtedly stimulate significant demand for process analytical technology (PAT)". PAT, aims to give a better scientific understanding of manufacturing process, which leads to knowledgebased production. This implies the monitoring of operating parameters, such as pressure, temperature or flow rates, but also physicochemical parameters as compositions, concentrations or particle sizes. 
Spectroscopic techniques, such as near infrared (NIR) spectroscopy, have proven useful in process control for several years and many uses attest of their high efficiency ${ }^{4-7}$. Near infrared spectroscopy offers a number of important advantages for inline analysis ${ }^{89}$. It is a nondestructive method, requiring minimal or no sample preparation, it is fast, and can be carried out without contact. Its implementation online is simple, and signal transport using optical fibers offers the advantage of relocating the analyzer away from hazardous areas.

Besides the practical advantages, an important reason for using NIR spectroscopy for process monitoring is its ability to supply versatile and multivariate information. Indeed, NIR spectroscopy is based on the principle of light interaction with matter allowing to obtain qualitative and quantitative physical and chemical information.

In combination with multivariate data analysis the spectral information can be correlated to product properties, as it has been done for a large number of applications in various fields. For instance:

- Qualitative monitoring can be done such as on wet agglomeration of wheat flour ${ }^{10}$, the conversion of a monomer during a polymerization reaction ${ }^{11}{ }^{12}$, to verify that a product is in specification by comparing it to reference spectra ${ }^{13}$ or to follow the structural modifications of a medium and detect the formation of a homogeneous gel as in the study of Mas et al. ${ }^{14}$ for example.

- In combination with multivariate data analysis NIR spectroscopy can be used for quantitative monitoring of various chemical concentrations such as glucose ${ }^{15,16}$, paracetamol ${ }^{13}$, lactose ${ }^{17}$, moisture content ${ }^{13}, 10$, or the quantification of heavy products in oil ${ }^{18}$, but also for physical properties like particle size ${ }^{11,19-23}$.

However, in heterogeneous samples, the interaction of light with matter is complex and spectra contain combinations of the effects of transmission, absorption and diffusion ${ }^{24}$. Generally, as the chemical information is sought, it is usual to correct for the scattering effects by means of empirical pretreatments ${ }^{25}$. But in the case of PAT, there is an interest to exploit the full potential of the spectral information and use both absorbance (which describes the chemistry) and scattering (which describes physicals properties as particle 
size, agglomeration, porosity) information. However heterogeneous systems cannot be fully characterized by a single measurement.

Recently, some studies focused on the use of multipoint measures for monitoring in situ heterogeneous systems. Scheibelhofer et al ${ }^{26}$ have combined multipoint NIR measurements with Monte Carlo simulation to understand the behavior of light in pharmaceutical tablets. Boiret and Chauchard ${ }^{27}$ have combined multipoint measurements in reflection with chemometrics tools, such as partial least squares (PLS) regression and principal component analysis (PCA), to predict sample hardness and active pharmaceutical ingredient (API) distribution within tablets respectively. The association of PLS regression with multipoint reflection measures has also shown good results for quantifying proteins and fat in milk ${ }^{28}$, to predict the fat and moisture content of meat ${ }^{29}$, for in-line moisture content analysis during freeze-drying ${ }^{30}$, or for in-situ estimation of concentration and particle size in colloidal suspensions ${ }^{31}$. In the study by Igne et al. ${ }^{32}$, a traditional singlepoint NIR measurement was compared with that of a spatially resolved spectroscopic (SRS) measurement for the determination of tablet assay by applying PLS regression also. Multipoint measurements have been shown to be more sensitive to tablet heterogeneity, even if the authors stated that multivariate multiblock analysis may further enhanced those.

Indeed, there would be an interest in associating multipoint measurements with specific multivariate data analysis methods, like common components specific weights analysis (CCSWA) ${ }^{33}$. CCSWA, also known as ComDim, is a multiblock analysis which makes it possible to analyze simultaneously several data matrices, considering them as blocks, and to extract the information that is common among them. Historically, this method was developed by Qannari et al. ${ }^{33-36}$ in order to analyze tables as part of sensory assessments. CCSWA has since been used to study samples analyzed on different instruments, to find relationships between tables and to discriminate samples on the basis of the global information included in all tables. For example Ammari et al. ${ }^{37}$ has studied the recognition of geological material by means of laser induced spectroscopy, coupled with three different spectrometers. They used CCSWA to analyze jointly the signals delivered by the three devices and to retrieve the best wavelength regions. In Kulmyrzaev et al. ${ }^{38}$, CCSWA 
was used to investigate changes in cheeses mixing rheology, infrared spectroscopy and front-face

fluorescence spectroscopy. This technique has also been successfully used to show that there is a correlation between particle size distribution and wheat flour NIR spectra ${ }^{39}$.

In the present paper, CCSWA coupled with multi-angle spectra measurements is proposed as a novel method to monitor in situ heterogeneous systems. Two heterogeneous systems are studied in this article. One where only the scattering varies, and another where the absorption and the scattering both change.

The silica precipitation reaction was chosen to illustrate the environment where only the scattering varies ${ }^{40}$. The manufacture of silica is a high-stakes industrial application because silica is a product with added value which can be used for many applications in various fields. The precipitation reaction stage is a crucial step in the silica production chain as the main characteristics of the final product depend on it. From these characteristics depend the uses of the silica produced. It is then essential to understand the mechanisms of this reaction and to follow it online.

The monitoring of processes to improve oil extraction was chosen to illustrate a situation where the absorption and the scattering both vary. The enhanced oil recovery (EOR) process involves injecting a saline solution containing surfactants into the rock. With this formulation, microemulsions are formed with the oil and extraction yields are improved. However, obtaining microemulsions is critical and depends on many parameters such as the nature of oil, the nature of the rocks or surfactants. Depending on these parameters, microemulsions with different physicochemical characteristics can be obtained. It is therefore essential to understand what happens in microemulsions, to identify the type of system created and to monitor in real time to ultimately optimize the process.

This paper is organized as follows. First, experimental set up are described for both studies. Then, the CCSWA is presented. Results are discussed in two parts: one from the silica precipitation study, and the other from the microemulsions study.

Materials and methods 
Study 1: Silica precipitation monitoring

\section{Material}

A specially designed probe (Sam-Flex, from Indatech, Chauvin Arnoux, France) was used for spectral measurements. It was composed of 5 positions for spectral measurements at 5 different angles, 3 in transmission at $150^{\circ}$ from the incoming beam, $170^{\circ}$ and $180^{\circ}$, and 2 in reflection at $30^{\circ}$ and $90^{\circ}$. The diagram of the probe is represented in Figure 1. The air-gap of the probe was $3 \mathrm{~mm}$ which correspond to the optical path. The probe was connected to a spectrometer (Hy-ternity, Indatech, France) composed of an NIR camera equipped with an InGaAs detector (340* 256 pixels) allowing the simultaneous measurement of all spectra. Spectral data were measured in the wavelength region from 950 to $1650 \mathrm{~nm}$ at $5 \mathrm{~nm}$ intervals.

\section{(i.e. "[insert Figure 1.]")}

Figure 1: Multiangle probe diagram (Sam-Flex, Indatech)

\section{Experimental setup}

Silica was prepared by neutralization of a sodium silicate solution (water glass) with sulfuric acid according to Equation 1 and Equation 2.

$$
\begin{aligned}
& \left(\mathrm{SiO}_{2}\right)_{x}\left(\mathrm{Na}_{2} \mathrm{O}\right)_{y}, \mathrm{nH}_{2} \mathrm{O}+y \mathrm{H}_{2} \mathrm{SO}_{4} \rightarrow \\
& x \mathrm{Si}(\mathrm{OH})_{4}+y \mathrm{Na}_{2} \mathrm{SO}_{4}+(y+n-2 x) \mathrm{H}_{2} \mathrm{O} \text { (hydrolysis) }
\end{aligned}
$$

$$
x \mathrm{Si}(\mathrm{OH})_{4} \rightarrow x \mathrm{SiO}_{2}+2 x \mathrm{H}_{2} \mathrm{O} \text { (polycondensation) }
$$


$\mathrm{nm}$ to form so-called elementary particles. These particles have coalesced into aggregates that are resistant to grinding or dispersion in a matrix. Aggregate dimensions can range between 50 and $500 \mathrm{~nm}$. At one specific moment in the reaction, the gel point, the entire volume has aggregated to form a continuous gel. Finally, because of a shear stress created continuously in the reaction medium, new aggregates of size between 0.2 and $40 \mu \mathrm{m}$ were formed ${ }^{41,42}$.

The synthesis (or batch) was carried out on a laboratory pilot in a perfectly stirred $25 \mathrm{~L}$ reactor. An aqueous solution of sodium sulfate was prepared and introduced in the reactor. Then, sulfuric acid and sodium silicate (provided by Solvay) were added alternatively according to the protocol established by the manufacturer (it is not possible to itemize). Their rate flows were regulated and automatized.

The probe was installed on a fast loop to monitor the precipitation reaction. The probe head was oriented so that the flow circulates in the gap. The experimental setup is represented in Figure 2.

\section{(i.e. "[insert Figure 2.]")}

Figure 2 : Experimental setup

Spectral acquisition

Spectra were acquired continuously with an integration time of $55 \mathrm{~ms}$ every $2 \mathrm{~s}$. The spectra recording was started at the first addition of reagents. It was stopped few minutes after the end of the reaction, when the product was stable.

The $\mathrm{I}_{0}$ reference was measured on air at angle $180^{\circ}$.

In order to compensate for the source spectrum and the internal response of the sensors, the transmission was calculated at each angle according to Equation 3: 
167

168

169

170

171

172

173

174

175

176

177

178

179

180

181

182

183

184
$S_{m}=\frac{I_{m}}{I_{0}}$

Equation 3

Where $\mathrm{I}_{0}$ is the intensity measured on air at angle $180^{\circ}$ and $\mathrm{I}_{\mathrm{m}}$ is the intensity measured on the sample at angle $\mathrm{m}$, with $m=30^{\circ}, 90^{\circ}, 150^{\circ}, 170^{\circ}$ and $180^{\circ}$.

Study 2: Micro emulsion monitoring

Material

A specially designed probe (Sam-Flex, from Indatech, Chauvin-Arnoux) was used for spectral measurements. It was composed of 5 positions at 5 different angles, 3 in transmission at $170^{\circ}$ from the incoming beam, $175^{\circ}$ and $180^{\circ}$, and 2 in reflection at $5^{\circ}$ and $10^{\circ}$. The air-gap of the probe was still 3 mm.

The probe was connected to the same spectrometer as before

Samples

The operating protocol applied to prepare the samples came from the work of Fukumito et al. ${ }^{43}$. In this publication, the different phases of the samples have been characterized, which makes it possible to know the state of the system when reproducing the exact same protocol: aqueous phase $\mathrm{W}$, or organic $\mathrm{O}$, micro emulsion water in oil $\mathrm{W} / \mathrm{O}$, or oil in water $\mathrm{O} / \mathrm{W}$, or oil and water $\mathrm{M}$.

First, solutions of SDBS (Sigma Aldrich) at $140 \mathrm{~g} / \mathrm{L}$ and $\mathrm{NaCl}$ (VWR chemicals) at $200 \mathrm{~g} / \mathrm{L}$ were prepared separately in flasks.

Then three different samples were made in $30 \mathrm{ml}$ glass bottles by successively adding:

- $2 \mathrm{~mL}$ of the SDBS solution

8 
- water and $\mathrm{NaCl}$ solutions. The volume of water and solution were calculated so that, in all samples, there was an aqueous phase volume of $13.58 \mathrm{ml}$ covering a set of salinity of 4, 32 and $64 \mathrm{~g}$ of $\mathrm{NaCl}$ / $\mathrm{L}$ of water.

- Also, $0.84 \mathrm{~mL}$ of isobutanol (Alfa Aesar) was added and carefully mixed.

- Finally, $13.58 \mathrm{~mL}$ of decane (Alfa Aesar) was added and carefully mixed.

Samples were mixed gently by turning the bottles upside down and left to be equilibrated for 1 month at room temperature.

For all 3 samples, the aqueous phases are in the lower part of the bottle and the organic phases in the upper part.

\section{Experimental setup}

A specific setup was developed to analyze the phase in line, in order to simulate the outflow of coreflood pilots ${ }^{44,45}$. Spectra were acquired off-contact through a quartz tube. The tube, with its internal diameter of 1 $\mathrm{mm}$ and its external diameter of $3 \mathrm{~mm}$, was put in the probe air-gap. Its upper end was crimped with a Swagelok fitting to seal. A non-beveled syringe needle was positioned at its lower end. The Swagelok fitting was connected to a $1 / 16^{\text {th }}$ inch PTFE tubing. The other end of the tubing was connected to a $50 \mathrm{ml}$ glass syringe. The syringe was installed on a syringe pump to make the sample flow. The setup is shown in Figure 3.

\footnotetext{
${ }^{\ddagger}$ A laboratory test in which a fluid or combination of fluids is injected into a sample of rock. Objectives include measurement of permeability, relative permeability, saturation change, formation damage caused by the fluid injection, or interactions between the fluid and the rock. A coreflood is typically used to determine the optimum development option for an oil reservoir and often helps evaluate the effect of injecting fluids specially designed to improve or enhance oil recovery.
}

9 
208 (i.e. "[insert Figure 3.]")

209 Figure 3 : Photograph of the experimental setup

210 Spectral acquisition

211 The syringe pump flow rate was set to $1 \mathrm{ml} / \mathrm{min}$ and maintained constant until $28 \mathrm{ml}$ of product was pumped.

212 Spectra were acquired continuously with an integration time of $1.65 \mathrm{~ms}$ every second.

213 The $\mathrm{I}_{0}$ reference was measured on the empty quartz tube for each angle.

214 In order to compensate for the light spectrum and the internal response of the sensors, the transmission was

215 calculated at each angle according to

216

217 Equation 4:

$218 S_{a}=\frac{I_{a}}{I_{0 a}}$

220 Equation 4

219

Where $\mathrm{I}_{\mathrm{a}}$ is the intensity measured on the sample through the tube at the angle $a$, and $\mathrm{I}_{\mathrm{oa}}$ is the intensity measured on the empty tube at the angle $a$, with $a=5^{\circ}, 10^{\circ}, 170^{\circ}, 175^{\circ}$ and $180^{\circ}$.

Multivariate analysis

Both sets of data were processed with MATLAB 2015b (The Mathworks, Natick, MA, USA).

Both times, the Savitzky-Golay function was performed to smooth spectra ( $2^{\text {nd }}$ order, 13 points, no derivative).

A CCSWA was applied on each data base. In both cases, the data bases were $k$ blocks of $n$ samples and $p$ wavelengths, here $p=201$ and $k=5$ which corresponded to the angle number.

The objective of CCSWA is to describe simultaneously the $k$ matrix $\mathbf{X}_{\mathbf{i}}$ observed for every $n$ samples. 10 
231 For each block $i$, the matrix $\mathbf{X}_{\mathbf{i}}$ has been centered by columns and the inertia matrix has been calculated as $232 \quad \mathbf{W}_{i}=\mathbf{X}_{\mathbf{i}} \cdot \mathbf{X}_{\mathbf{i}}^{\prime}$

233 The matrix $\mathbf{W}_{\mathbf{i}}$ reflects the dispersion of the samples in the data space, for the block i. Since the number of 234 samples was the same for all blocks, all $\mathbf{W}_{\mathrm{i}}$ matrices had the same size (nxn). The common dimensions of all matrix was calculated iteratively according to ${ }^{35,46:}$

$$
\mathbf{W}_{i}=\sum_{\operatorname{dim}=1}^{d} \lambda_{d i m}^{(i)} \cdot \mathbf{q}_{d i m} \cdot \mathbf{q}_{d i m}^{\prime}+\mathbf{R}_{i} \quad 238 \text { Equation } 5
$$

Where $d$ is the number of dimensions which has to be fixed, $\lambda_{\text {dim }}^{(i)}$ is the specific weight (='salience') of the matrix $\mathbf{X}_{\mathbf{i}}$ in the construction of the common component $\mathbf{q}_{\text {dim }}$ in the dimension $\operatorname{dim}$, and $\mathbf{R}_{\mathbf{i}}$ the residual matrix of $\mathbf{X}_{\mathbf{i}}$. So, each common component $\mathbf{q}_{\text {dim }}$ is weighted by a scalar $\lambda_{\text {dim }}^{(i)}$ reflecting the contribution of the matrix $\mathbf{X}_{\mathrm{i}}$ in the construction of $\mathbf{q}_{\mathrm{dim}}{ }^{47,36}$.

The method consists in determining a common space for all $k$ blocks, with each matrix having a specific contribution ("salience"), $\lambda_{d i m}^{(i)}$, to the definition of each dimension, $\mathbf{q}_{\mathrm{dim}}$, of this common space by maximizing the variance common to all blocks. Global scores, individual scores and loadings, and saliences in the construction of common dimensions were

Results and discussions

251 Spectral interpretation 
Table 1: Contribution of angles in the construction of common dimensions

\begin{tabular}{|l|c|c|c|c|}
\cline { 2 - 5 } \multicolumn{1}{c|}{} & Dimension 1 & Dimension 2 & Dimension 3 & Residuals \\
\hline $30^{\circ}$ & $83.2 \%$ & $10.8 \%$ & $6.0 \%$ & $0.1 \%$ \\
\hline $90^{\circ}$ & $97.6 \%$ & $0.5 \%$ & $1.9 \%$ & $0.1 \%$ \\
\hline $150^{\circ}$ & $99.3 \%$ & $0.6 \%$ & $0.1 \%$ & $0.05 \%$ \\
\hline
\end{tabular}
optical path of $3 \mathrm{~mm}$ is too important to exploit the absorption band at $1450 \mathrm{~nm}$.

\section{(i.e. "[insert Figure 4.]")}

Figure $4:$ Spectra $\mathrm{I} / \mathrm{I}_{0}$ at the five angles throughout the batch

\section{CCSWA}

Study of contributions common dimensions (= common components)

Figure 4 shows spectra $S_{\mathrm{m}}$ acquired during the silica precipitation reaction. For all angles the same transmission profiles are observed. These are due to water absorption bands ${ }^{48}$. The second overtone of the $\mathrm{OH}$ stretching band $\left(3 v_{1,3}\right)$ is at $970 \mathrm{~nm}$, the combination of the first overtone of the $\mathrm{OH}$ stretching band and the $\mathrm{OH}$ bending band $\left(2 v_{1,3}+v_{2}\right)$ are at $1190 \mathrm{~nm}$, and the first overtone of the $\mathrm{OH}$ stretching band $\left(2 v_{1,3}\right)$ is at $1450 \mathrm{~nm}$. The transmission profiles are stuck to the baseline when the signal is saturated in absorption. The

A CCSWA was applied on the batch. Table 1 presents the contributions of angles in the construction of 


\begin{tabular}{|l|c|c|c|c|}
\hline $170^{\circ}$ & $4.7 \%$ & $95.2 \%$ & $0.1 \%$ & $0.02 \%$ \\
\hline $180^{\circ}$ & $90.2 \%$ & $9.2 \%$ & $0.6 \%$ & $0.1 \%$ \\
\hline Variance & $69.6 \%$ & $30 \%$ & $0.4 \%$ & \\
\hline
\end{tabular}

268

The explained variance and the residuals of the sum of the contributions of angles to each dimension show that three dimensions are sufficient to explain almost the entire variance of the dataset. Calculations for 2 and 4 dimensions were carried out but was not considered as relevant.

The first two dimensions carry the major part of variance. All angles but $170^{\circ}$ participate in the construction of the first dimension. Symmetrically, the second dimension mainly relies on the $170^{\circ}$ angle. Dimension 3 is built mainly by the angle at $30^{\circ}$ and to a lesser extent at $90^{\circ}$.

Study of common scores across all dimensions

CCSWA also provided overall scores, they are plotted against time for the three dimensions in Figure 5.

\section{(i.e. "[insert Figure 5.]")}

Figure 5 : Common scores for three common dimensions according to time. The dotted lines correspond to the modifications of the process, the star and the numbered strips correspond to product evolutions

Overall, two main types of events are observed at each dimension on Figure 5. 
On the one hand, sharp slope changes are observed in all three dimensions. They are highlighted on the figure by dashed lines. Thanks to the readings of the automaton from the pilot, it was possible to link these slope changes to process actions: such as, adding or stopping sodium silicate or sulfuric acid.

On the other hand, general slopes with minima and / or maxima are observed on the dimensions. These slopes modifications are not linked to instants of the reaction but to changes in the product. These are shown in Figure 5 by the green star and the numbered frieze in the lower part. The number represent the main steps of product evolution (although they are not fixed and probably are entangled as product is gradually evolving from one stage to the next). They are determined based on the knowledge acquired by the manufacturer and to the literature ${ }^{49-53}$. Step 1 corresponds to elementary particles aggregation. At one specific moment in the reaction, represented by the green star in Figure 5, the entire volume aggregates to form a continuous gel. It is called the gel point. At this point, the physical structure of the medium instantly changes. The moment the gel point occurs was validated by a turbidity monitoring retrospectively. During step 2, because of the shear stress created continuously in the reaction medium, aggregates are formed. Then during step 3, agglomerates rearrange and become denser, their internal structure is consolidated. During step 4 , only one of the 2 reagents is added which dilutes the medium. Finally, the medium remains constant in the last section.

\section{Study of common scores}

For all dimensions the scores profile is constant until the gel point, around $800 \mathrm{AU}$, with more or less noise. This profile is linked to the fact that at the beginning of the reaction the medium is almost limpid and contains few particles (this had been observed in a previous laboratory study ${ }^{40}$ ). Particles, if present had a diameter smaller than tens of nanometers. For the studied wavelengths, the scattering that can be generated by these particles is very small, and is not detected. 
The scores profile of the second dimension show a maximum. A very strong increase is observed simultaneously to the gel point up to about $1200 \mathrm{AU}$. In the rest of the batch, the scores decrease until they stabilize at the end of the reaction. Table 1 which gives the contribution of angles in the construction of dimensions showed the specialization of the angle at $170^{\circ}$ for the construction of the second dimension. It turns out that this angle is sensitive to serpentine photons. These photons are the ones that have slightly scattered while maintaining their rectilinear trajectory. It is likely that this dimension is sensitive to the simple scattering in the medium. This would be in line with the scores profile at the gel point and during shearing. Indeed, during the gel formation, the scores increase because the gelation makes the whole sapphire window and reaction medium's refractive index more homogeneous, creating an optical continuity of the medium. The gel conducts the light forward. Then, when shearing the gel, more and more particles are generated. There are therefore fewer serpentine photons and therefore less simple scattering, hence the decrease in scores.

This sensitivity to the simple scattering has been observed on the loadings too. In the Figure 6 the loadings obtained at $170^{\circ}$ in the second component and the average spectrum of the batch are represented.

\section{(i.e. "[insert Figure 6.]")}

Figure 6: Average spectrum of the batch and loadings obtained for the angle $170^{\circ}$ in the second dimension

Two scattering consequences can be observed. From 1350 to $1600 \mathrm{~nm}$, an absorption band is observed. It correspond to water absorption band. This band is related to the optical path of the photon. In a previous study ${ }^{40}$, the absorption was considered as constant during the reaction. Therefore, a variation of the height of 
the band is related to the increase of the optical path and thus to the scattering of the photons ${ }^{54}$. A higher absorption makes the spectrum lower in this region. Consequently, the higher the diffusion, the longer the optical path and the lower the spectrum. When multiplied by the positive peak of the loading, it produces a lower score. Then, a slope is observed on loadings from 950 to $1350 \mathrm{~nm}$. This part of the loading calculates the general slope of the spectrum between 950 and $1350 \mathrm{~nm}$. It appears as a rotation of the average spectrum. This slope is connected to the scattering too ${ }^{55,56}$. The higher the diffusion, the steeper the slope. Thus high diffusion leads to low scores. Consequently, the loadings are sensitive to the diffusion of light. High scores correspond to low diffusion, such as after the gel point, when the gel acts as a light guide.

Finally, thanks to the scores of the second dimension represented in Figure 5, it is possible to detect the gel point very precisely and accurately. This aspect is very important as this gel point is critical for determining the products future qualities. It shows that the second dimension is very informative and necessary to ensure the monitoring of the reaction. The CCSWA therefore demonstrated the specific interest of the $170^{\circ}$ angle to monitor this manufacturing process.

The score profile of the first dimension increases throughout the reaction. With regard to Table 1, all angles except the one at $170^{\circ}$ participate in its construction. These angles do not detect the same type of photons. Ballistic photons are detected at $180^{\circ}$, backscattered photons at $30^{\circ}$ and $90^{\circ}$ and forward scattered photons at $150^{\circ}$. This first dimension seems to represent the general scattering of the reaction medium, that is to say the global propagation of photons without distinction between ballistic and scattered photons. Therefore we assume that the multiple scattering in the reaction medium is represented in the first dimension

The scores of the third dimension show a minimum and a maximum. The decrease to reach the minimum takes place in the same period as the growth to reach the maximum of the second dimension, just after the gel 
point. At the opposite, during shearing, the scores of the third dimension increase to reach a maximum when those of the second dimension decrease. Lastly, the scores decrease until they stabilize at the end of the reaction. The third dimension probably represents the backscattered photons since the angles at $30^{\circ}$ and $90^{\circ}$ contribute mainly to its construction (see Table 1). At the gel point, it has been said previously that the optical continuity of the sapphire window with the reaction medium conducts to better light transmission. More photons propagate forward, fewer backscatter. The decrease in the amount of backscattered photons results in a decrease in the profile of the scores in the third dimension. CCSWA tends to consider the optical system as a distribution of a total amount of photons. That is why, compared to the totality of photons at the five angles, the number of photons detected at angles $30^{\circ}$ and $90^{\circ}$ decreases. Then during shearing, the number of particles increases while their size decreases. This induces the increase of scores in the third dimension and the decrease of scores in the second dimension. When the medium is sheared further, there is a decrease in serpentine photons and an increase in backscattered multi-scattered photons. Shear causes increased scattering of particles and the decrease in their size influences the direction of scattering. This is in agreement with the theory ${ }^{13}$ demonstrating that the smaller the particle size, the less photons will tend to scatter forward. The final decrease in the third dimension scores takes place in the last stage of the precipitation reaction. Parallel to the formation of new particles in the medium, aggregates fill the already existing agglomerates. They rearrange themselves, their internal structure is modified and more compact. This modifies, both the specific surface area and thus the micro porosity and the number of hydrogen bonds ${ }^{1}$. These phenomena reduce the amount of light backscattered, hence the decrease in scores in the third dimension. Even if at the same time, the multiple scattering continues to increase as shown by the scores of the first dimension.

The third dimension seems to be clearly related to the direction of the scattering of photons in the medium during the reaction. 
Study 2: Micro emulsion monitoring

Samples

Solutions which were obtained are represented in the Figure 7.

\section{(i.e. "[insert Figure 7.]")}

Figure 7 : Photograph of the 3 samples for the micro emulsion application

The sample's number corresponds to the salinity of the aqueous phase (always the lower phase in the vials).

The different phases present in these 3 samples can be grouped into five classes, as described in Table 2 .

Table 2: Phase classification

\begin{tabular}{|c|c|c|c|}
\hline Salinity of the aqueous phase & 64 & 32 & 4 \\
\hline Organic phases (upper) & 1 & 2 & 2 \\
\hline Aqueous phases & 4 & 4 & 3 \\
(lower) & & & \\
\hline Middle phases & $\mathrm{NC}$ & 5 & $\mathrm{NC}$ \\
\hline
\end{tabular}

386

387 Class 1 contains the organic phase where there is a micro emulsion of water in oil (W/O), as in sample 64,

388 which can be described as a Winsor II in the literature ${ }^{43}$.

389 Class 2 are the organic phases consisting only of decane $(\mathrm{O})$, as in samples 32 and 4. 
390 Class 3 is the aqueous phase where there is a micro emulsion of oil in water $(\mathrm{O} / \mathrm{W})$, as in sample 4 , which 391 can be described as a Winsor I.

Class 4 are the aqueous phases consisting only of salt water with different concentrations of salt, as in samples 64 and 32 .

Class 5 is the middle phase (M) where there is a micro emulsion of water and oil which are only present in Winsor III samples.

The visual inspection of the phases (Figure 7) shows that Class 2 phases are transparent and colorless. The Class 1 and 3 phases seem slightly cloudy. For Class 4, sample 64 phase is transparent and colorless, but sample 32 is whitish in its upper part and tends to be transparent in the lower part. Finally, the Class 5 phase is whitish and cloudy throughout.

The cloudiness is probably due to the presence of micro emulsions. The medium appears whiter the more oil is dispersed in the water. Therefore, micro emulsions probably generate scattering. The aqueous phase of sample 32, should belong to a Class 4 and therefore not contain oil. This sample's whitish gradient is probably due to a transition between the $\mathrm{O} / \mathrm{W}$ micro emulsion and the pure aqueous phase.

\section{Spectral interpretation}

For a spectral interpretation of classes, only the mean absorption spectrum of each class for the $180^{\circ}$ angle is represented in Figure 8.

\section{(i.e. "[insert Figure 8.]")}

Figure 8 : Mean absorption spectra of classes 
Two groups can be observed, separated by an important absorption difference at $1450 \mathrm{~nm}$ and their baseline. A first group contains Classes 1 and 2 and a second group the Classes 3, 4 and 5.

The first group contains organic phases. The absorption bands of decane are found at $1208 \mathrm{~nm}$ and $1408 \mathrm{~nm}$. The second overtone of symmetrical and asymmetric elongations of $\mathrm{CH}_{2}$ and $\mathrm{CH}_{3}$ groups is observed at 1208 $\mathrm{nm}$, while the absorption band at $1408 \mathrm{~nm}$ corresponds to the first overtone of the combination between the symmetric and asymmetric elongations of the $\mathrm{CH}$ bonds in the $\mathrm{CH}_{2}$ and $\mathrm{CH}_{3}$ groups and the deformations of the $\mathrm{C}-\mathrm{H}$ bonds. Between the mean spectrum of Class 1 and the mean spectrum of Class 2, an absorption difference is observed at the band at $1408 \mathrm{~nm}$. This is the absorption of water present in the Class 1 .

By observing the absorption bands of the mean spectra of the second group, the water absorption bands at $970 \mathrm{~nm}, 1190 \mathrm{~nm}$ and $1450 \mathrm{~nm}$ are found. Their identification was made for the previous application. Within this group, the mean spectrum of Class 5 differs from the other two classes. At $1208 \mathrm{~nm}$ an absorption band is observed, which is assigned as the absorption band of decane. The absorption at $1408 \mathrm{~nm}$ is not observable, as it is hidden under the large water band at $1450 \mathrm{~nm}$. In this wavelength range, the absorption of water is much stronger than the absorption of decane. Between the mean spectra of Class 3 and 4 , a slight difference is observed on the $1450 \mathrm{~nm}$ band. The band seems slightly shifted to the left for the Class 4 spectrum. This may be due to the presence of salt in the Class 4 phases which could create a slight band shift ${ }^{14,48}$. In addition, the baseline of the mean spectrum of Class 4 is higher than that of the mean spectrum of Class 3. The presence of a baseline is one of the consequences of photon scattering ${ }^{40}$. This is in line with the visual observation of the samples in Figure 7, where a whitish appearance of Class 4 was observed, probably related to scattering effects. 
Table 3: Contribution of angles in the construction of common dimensions

\begin{tabular}{|l|c|c|c|c|}
\cline { 2 - 5 } \multicolumn{1}{c|}{} & Dimension 1 & Dimension 2 & Dimension 3 & Residuals \\
\hline $5^{\circ}$ & $68.2 \%$ & $26.4 \%$ & $5.4 \%$ & $0 \%$ \\
\hline $10^{\circ}$ & $69.0 \%$ & $25.5 \%$ & $5.5 \%$ & $0 \%$ \\
\hline $170^{\circ}$ & $91.3 \%$ & $8.2 \%$ & $0.5 \%$ & $0 \%$ \\
\hline $175^{\circ}$ & $85.7 \%$ & $10.9 \%$ & $3.4 \%$ & $0 \%$ \\
\hline $180^{\circ}$ & $86.5 \%$ & $12.4 \%$ & $1.1 \%$ & $0 \%$ \\
\hline Variance & $87.1 \%$ & $12.4 \%$ & $0.5 \%$ & \multirow{2}{*}{} \\
\hline
\end{tabular}
this study is to determine if there is a gain to associate multi-angle analysis with CCSWA.

\section{CCSWA}

Study of contributions

A CCSWA was applied on all five classes of all 5 angles spectra.

Table 3 presents the contribution of angles in the construction of common dimensions.

The observation of mean spectra shows that it is possible to identify almost all classes by NIR spectroscopy.

For each class, differences are observable at water and decane absorption bands. The goal for the next part of 
The explained variance shows that three dimensions are sufficient to explain almost the entire variance of the dataset. (With regard to residuals, we see that the contributions of each angle are distributed in the three dimensions.)

All angles participate in the construction of the first dimension and predominantly those in transmission. Contrary to the second dimension where the contributions of reflection angles are predominant. The third dimension, is also built mainly by angles $5^{\circ}$ and $10^{\circ}$ with a contribution of the angle at $175^{\circ}$ to a lesser extent. The third dimension represents very little variance in comparison with the first two.

\section{Study of common scores across all dimensions}

CCSWA provided common scores. The scores obtained for each class were put end to end, to be able to compare them. The 2 phases of Classes 2 and 4 being identical, only the scores of the binary samples were represented. They are plotted according to the class for the three dimensions in Figure 9.

\section{(i.e. "[insert Figure 9.]")}

Figure 9 : Common scores for three common dimensions according to the class

Overall, common scores for the three dimensions show that each class has a different profile. It is already possible to say that the CCSWA makes it possible to separate the different classes.

The common scores of the first dimension (shown in blue) separate three groups of classes. Classes 1 and 2 , then Classes 3 and 4 and finally Class 5. Classes 1 and 2 have negative scores, while Classes 3 and 4 have positive scores. Those in Class 5 are positive but almost null. This dimension seems related to the presence of water in the samples. Indeed Classes 1 and 2, having negative scores, are organic phases and contain little or no water, while Classes 3 and 4, which have positive scores, are aqueous phases. Finally, Class 5 contains 22 
water and oil in almost equal proportions. This observation of the scores relate to the observations made in Figure 8 representing the mean spectra of Classes at $180^{\circ}$. Moreover, this is in line with the individual loadings of the transmission angles, shown in Figure 10, which strongly resemble the absorption spectrum of water.

\section{(i.e. "[insert Figure 10.]")}

Figure 10: Individual loadings of transmission angles to the first dimension

The common scores of the second dimension allow to separate the classes within the groups identified in the first dimension. Thanks to the second dimension, Class 1 differs from Class 2, and Class 3 differs from Class 4. Class 5 remains separate in this dimension as well. Class 5 scores are the most important, followed by Class 4, Class 3 and Class 1, and Class 2 scores, which are the lowest. This order is very close to the order of the baseline of the mean absorption spectra of the phases shown in Figure 8 . The second dimension probably is a general indication of the scattering in general of photons ${ }^{24}$.

The common scores of the third dimension bring together two groups of classes that had never been grouped before. A first group consists of Classes 2, 3 and 5, and a second group consists of Classes 1 and 4.

In the first group, the commonality between classes is the presence of decane. For Classes 3 and 5, decane is dispersed in water. In Class 2, only decane is present.

In the second group, the commonality between classes is the presence of water. In Class 1, water is dispersed in decane and in Class 4, only water is present.

The third dimension is mainly constructed by the angles at $5^{\circ}$ and $10^{\circ}$ according to the Table 3 . 
To try to interpret the profile of the scores, the individual loadings of the angles $5^{\circ}$ and $10^{\circ}$ were represented in the Figure 11.

\section{(i.e. "[insert Figure 11.]")}

Figure 11: Individual loadings of angles at $5^{\circ}$ and $10^{\circ}$ of the third dimension

Although these loadings are very noisy, information can still be exploited. Around $1660 \mathrm{~nm}$, the beginning of a hollow is observed. This hollow is the beginning of the absorption band of the first harmonic symmetrical and asymmetrical elongations of the $\mathrm{CH}_{2}$ and $\mathrm{CH}_{3}$ groups of decane. This decane absorption band is much larger than those at $1208 \mathrm{~nm}$ and $1408 \mathrm{~nm}$ and is even greater than the water absorption. It is probably the presence of this band, which makes it possible to differentiate the groups in the profile of the common scores in the third dimension. It may be that the third dimension is related to the presence of decane as a scattering product.

\section{Conclusions and perspectives}

This study, implemented on two industrial applications, shows the interest of multi-angle spectral measurements and their coupling to a Common Component and Specific Weight Analysis for monitoring processes.

In the case of monitoring the precipitation of silica, where only light diffusion effect varies, CCWAS associated with multi-angle NIR measurements has demonstrated the complementarity of the angles and its interest for process monitoring. Thanks to the scores obtained by the CCSWA, the actions inherent to the progress of the process could be identified by the different slopes breaks in the dimensions scores plot. Each physicochemical modification of the reaction medium induced scattering phenomena ${ }^{57}$ which resulted in 24 
curvatures, maxima and minima depending on the dimensions observed. It has been possible to identify a multiple scattering regime, a simple scattering regime, and the preferred direction of light propagation in the medium during the advancement of the precipitation reaction. CCSWA also confirmed the complementarity of the angles for monitoring this reaction and the interest of multi-angle measurements. This first study shows the interest of using this type of multivariate analysis on multi-angle spectral data. Regarding the preliminary study about micro emulsion measurements, where both absorption and scattering vary, CCSWA has allowed unequivocal identification of the different phases. At each dimension, different phenomena have been observed. This is a gain for users because through this identification, it will be possible to know in real time the type of medium obtained (aqueous phase $\mathrm{W}$, or organic $\mathrm{O}$, micro emulsion water in oil $\mathrm{W} / \mathrm{O}$, or oil in water $\mathrm{O} / \mathrm{W}$, or oil and water $\mathrm{M}$ ) without sampling and with only one analytical technique. This monitoring is a real asset in the optimization of operational parameters on R \& D pilots.

Overall, the tests implemented have shown that it is very interesting to couple multi-angle measurements with common component and specific weight analysis. Initially, this CCSWA tool was developed to analyze data from sensory analyzes of different dimensions ${ }^{47}$. But, the results obtained allowed reversion to the physicochemical information of the mediums, starting from multi-angle spectral measurements having the same dimension. Nevertheless, it would be interesting to explore the results of other existing tools for multiarray multivariate analysis, and even to develop a specific tool for the analysis of multi-angle spectral data. This study also shows the gain of monitoring in line processes, even when complex medium are involved.

\section{Conflict of interest}


The authors declare that they have no conflict of interest

\section{References}

1. R.W. Kessler, "Perspectives in process analysis", J. Chemometrics, 27, 11 (2013).

2. Food and Drug Administration, Guidance for Industry. Process Validation: General Principles and Practices (Janvier 2011).

3. R. W. Kessler, K. Rebner and W. Kessler, eds., Multi-Modal-Spectroscopy and Multivariate Data Analysis as a Tool for Non-Invasive Process Analysis (2013).

4. J. Workman, "A review of process near infrared spectroscopy: 1980-1994”, Journal of Near Infrared Spectroscopy, 1 (1993).

5. L.L. Simon, H. Pataki, G. Marosi, F. Meemken, K. Hungerbühler, A. Baiker, S. Tummala, B. Glennon, M. Kuentz, G. Steele, H.J.M. Kramer, J.W. Rydzak, Z. Chen, J. Morris, F. Kjell, R. Singh, R. Gani, K.V. Gernaey, M. Louhi-Kultanen, J. O’Reilly, N. Sandler, O. Antikainen, J. Yliruusi, P. Frohberg, J. Ulrich, R.D. Braatz, T. Leyssens, M. von Stosch, R. Oliveira, R.B.H. Tan, H. Wu, M. Khan, Des O'Grady, A. Pandey, R. Westra, E. Delle-Case, D. Pape, D. Angelosante, Y. Maret, O. Steiger, M. Lenner, K. Abbou-Oucherif, Z.K. Nagy, J.D. Litster, V.K. Kamaraju and M.-S. Chiu, “Assessment of Recent Process Analytical Technology (PAT) Trends: A Multiauthor Review", Org. Process Res. Dev., 19, 1 (2015). 6. J. Workman, B. Lavine, R. Chrisman and M. Koch, "Process analytical chemistry", Analytical chemistry, 83, 12 (2011).

7. Process column, ed., Process Spectroscopy, moving spectroscopy from lab to line (2003).

8. Y. Ozaki, “Near-Infrared Spectroscopy: Its Versatility in Analytical Chemistry”, Anal. Sci., 28, 6 (2012).

9. D.A. Burns and E.W. Ciurczak, eds., Handbook of near-infrared analysis. CRC Press, USA (2008).

10. A. Ait Kaddour and B. Cuq, "In line monitoring of wet agglomeration of wheat flour using near infrared spectroscopy", Powder technology, 190, 1-2 (2009). 26 
11. W.K. Silva, D.L. Chicoma and R. Giudici, “In-situ real-time monitoring of particle size, polymer, and monomer contents in emulsion polymerization of methyl methacrylate by near infrared spectroscopy", Polym Eng Sci, 51, 10 (2011).

12. N. Heigl, C.H. Petter, M. Rainer, M. Najam-ul-Haq, R.M. Vallant, R. Bakry, G.K. Bonn and C.W. Huck, “Near Infrared Spectroscopy for Polymer Research, Quality Control and Reaction Monitoring", Journal of Near Infrared Spectroscopy, 15, 5 (2017).

13. Y. Roggo, P. Chalus, L. Maurer, C. Lema-Martinez, A. Edmond and N. Jent, "A review of near infrared spectroscopy and chemometrics in pharmaceutical technologies", Journal of pharmaceutical and biomedical analysis, 44, 3 (2007). 14. S. Mas, R. Bendoula, G. Agoda-Tandjawa, A. de Juan and J.-M. Roger, "Study of time-dependent structural changes of laponite colloidal system by means of near-infrared spectroscopy and hybrid hard- and soft-modelling multivariate curve resolution-alternating least squares", Chemometrics and Intelligent Laboratory Systems, 142 (2015).

15. M. Sandor, F. Rüdinger, D. Solle, R. Bienert, C. Grimm, S. Groß and T. Scheper, "NIR-spectroscopy for bioprocess monitoring \& control", BMC Proc, 7, Suppl 6 (2013).

16. H. Xu, B. Qi, T. Sun, X. Fu and Y. Ying, "Variable selection in visible and near-infrared spectra: Application to online determination of sugar content in pears", Journal of Food Engineering, 109, 1 (2012).

17. H. Huang, H. Yu, H. Xu and Y. Ying, "Near infrared spectroscopy for on/in-line monitoring of quality in foods and beverages: A review", Journal of Food Engineering, 87, 3 (2008).

18. J. Laxalde, C. Ruckebusch, O. Devos, N. Caillol, F. Wahl and L. Duponchel, “Characterisation of heavy oils using near-infrared spectroscopy: optimisation of pre-processing methods and variable selection", Analytica chimica acta, 705, 1-2 (2011).

19. L.K.H. Bittner, N. Heigl, C.H. Petter, M.F. Noisternig, U.J. Griesser, G.K. Bonn and C.W. Huck, “Near-infrared reflection spectroscopy (NIRS) as a successful tool for simultaneous identification and particle size determination of amoxicillin trihydrate", Journal of pharmaceutical and biomedical analysis, 54, 5 (2011).

27 
20. E. Prendergast, H. Abe, M. Aburada and M. Otsuka, “A Non-Destructive Method of Predicting the Particle Size of the Bulk Drug Powder in an Acetaminophen Suppository by Near-Infrared Spectroscopy", Journal of Near Infrared Spectroscopy, 20, 2 (2012).

21. A.J. O'Neil, R.D. Jee and A.C. Moffat, "Measurement of the percentage volume particle size distribution of powdered microcrystalline cellulose using reflectance near-infrared spectroscopyElectronic supplementary information (ESI) available: Particle size and spectral data. See http://www.rsc.org/suppdata/an/b3/b307263k", Analyst, 128, 11 (2003).

22. R. Liu, L. Li, W. Yin, D. Xu and H. Zang, "Near-infrared spectroscopy monitoring and control of the fluidized bed granulation and coating processes-A review", International journal of pharmaceutics, 530, 1-2 (2017).

23. M.C. Pasikatan, J.L. Steele, C.K. Spillman and E. Haque, "Near Infrared Reflectance Spectroscopy for Online Particle Size Analysis of Powders and Ground Materials", Journal of Near Infrared Spectroscopy, 9, 3 (2017). 24. R. Bendoula, Développements optiques pour améliorer la mesure spectrale des milieux biologiques complexes: applications agro-environnementales. Mémoire d'habilitation à diriger des recherches (2016).

25. A. Gobrecht, J.-M. Roger and V. Bellon-Maurel, "Major Issues of Diffuse Reflectance NIR Spectroscopy in the Specific Context of Soil Carbon Content Estimation", in: Advances in agronomy, Ed by D.L. Sparks, Academic Press, an imprint of Elsevier, San Diego, CA, pp. 145-175 (2014).

26. O. Scheibelhofer, P.R. Wahl, B. Larchevêque, F. Chauchard and J.G. Khinast, "Spatially Resolved Spectral Powder Analysis: Experiments and Modeling", Applied spectroscopy (2018).

27. M. Boiret and F. Chauchard, "Use of near-infrared spectroscopy and multipoint measurements for quality control of pharmaceutical drug products", Analytical and bioanalytical chemistry, 409, 3 (2017).

28. A. Bogomolov, V. Belikova, V. Galyanin, A. Melenteva and H. Meyer, "Reference-free spectroscopic determination of fat and protein in milk in the visible and near infrared region below 1000nm using spatially resolved diffuse reflectance fiber probe", Talanta, 167 (2017). 

29. Y. Dixit, M.P. Casado-Gavalda, R. Cama-Moncunill, P.J. Cullen and C. Sullivan, "Challenges in Model Development for Meat Composition Using Multipoint NIR Spectroscopy from At-Line to In-Line Monitoring”, Journal of food science, 82, 7 (2017).

30. A. Kauppinen, M. Toiviainen, M. Lehtonen, K. Järvinen, J. Paaso, M. Juuti and J. Ketolainen, “Validation of a multipoint near-infrared spectroscopy method for in-line moisture content analysis during freeze-drying", Journal of pharmaceutical and biomedical analysis, 95 (2014).

31. Y.-C. Chen, D. Foo, N. Dehanov and S.N. Thennadil, "Spatially and angularly resolved spectroscopy for in-situ estimation of concentration and particle size in colloidal suspensions", Analytical and bioanalytical chemistry, 409, 30 (2017).

32. B. Igne, S. Talwar, H. Feng, J.K. Drennen and C.A. Anderson, "Near-Infrared Spatially Resolved Spectroscopy for Tablet Quality Determination”, Journal of pharmaceutical sciences, 104, 12 (2015).

33. M. Hanafi and E.M. Qannari, "Nouvelles propriétés de l'analyse en composantes communes et poids spécifiques", Journal de la Société Française de Statistique, 149, 2 (2008).

34. E.M. Qannari, I. Wakeling, P. Courcoux and H.J. MacFie, "Defining the underlying sensory dimensions", Food Quality and Preference, 11, 1-2 (2000).

35. E.M. Qannari, P. Courcoux and E. Vigneau, "Common components and specific weights analysis performed on preference data", Food Quality and Preference, 12, 5-7 (2001).

36. M. Hanafi, G. Mazerolles, E. Dufour and E.M. Qannari, “Common components and specific weight analysis and multiple co-inertia analysis applied to the coupling of several measurement techniques", J. Chemometrics, 20, 5 (2006).

37. F. Ammari, L. Bassel, C. Ferrier, D. Lacanette, R. Chapoulie and B. Bousquet, "Multi-block analysis coupled to laserinduced breakdown spectroscopy for sorting geological materials from caves", Talanta, 159 (2016). 
38. A. Kulmyrzaev, É. Dufour, Y. Noël, M. Hanafi, R. Karoui, E.M. Qannari and G. Mazerolles, "Investigation at the molecular level of soft cheese quality and ripening by infrared and fluorescence spectroscopies and chemometricsrelationships with rheology properties", International Dairy Journal, 15, 6-9 (2005).

39. J. Pram Nielsen, D. Bertrand, E. Micklander, P. Courcoux and L. Munck, "Study of NIR spectra, particle size distributions and chemical parameters of wheat flours: a multi-way approach", Journal of Near Infrared Spectroscopy, 9 (2001).

40. M. Rey-Bayle, R. Bendoula, S. Henrot, K. Lamiri, F. Baco-Antoniali, N. Caillol, A. Gobrecht and J.-M. Roger, "Potential of vis-NIR spectroscopy to monitor the silica precipitation reaction", Analytical and bioanalytical chemistry, 409, 3 (2017).

41. I. Boudimbou, Mécanismes élémentaires de dispersion de charges de silice dans une matrice élastomère, Paris (2011).

42. Deka MOUSSA RAGUEH, Filtration de silices précipitées: mise en évidence des relations entre propriétés macroscopiques et échelles locales caractéristiques dans les dépôts, Toulouse (2011).

43. A. Fukumoto, C. Dalmazzone, D. Frot, L. Barré and C. Noïk, "Investigation on Physical Properties and Morphologies of Microemulsions formed with Sodium Dodecyl Benzenesulfonate, Isobutanol, Brine, and Decane, Using Several Experimental Techniques", Energy Fuels, 30, 6 (2016).

44. s. donertas, "Core Flooding Sytem", http://www.corelab.com/sanchez/enhanced-oil-recovery/cfs-700.

45. S. Shaddel, M. Hemmati, E. Zamanian and N. Moharrami, "Core flood studies to evaluate efficiency of oil recovery by low salinity water flooding as a secondary recovery process", Journal of Petroleum Science and Technology, 4 (2014).

46. E. Dubin, M. Spiteri, A.-S. Dumas, J. Ginet, M. Lees and D.N. Rutledge, “Common components and specific weights analysis: A tool for metabolomic data pre-processing", Chemometrics and Intelligent Laboratory Systems, 150 (2016). 47. D.N. Rutledge, Extensions of Common Component and Specific Weight Analysis for applications in Chemometrics, AgroParisTech (2013). 
48. H. Büning-Pfaue, "Analysis of water in food by near infrared spectroscopy", Food Chemistry, 82, 1 (2003).

49. J. Schlomach and M. Kind, "Investigations on the semi-batch precipitation of silica", Journal of Colloid and Interface Science, 277, 2 (2004).

50. J. Baldyga, M. Jasinska, K. Jodko and P. Petelski, Precipitation of amorphous colloidal silica from aqueaous solutions (2012).

51. J. Gregory, "Monitoring particle aggregation processes", Advances in colloid and interface science, 147-148 (2009).

52. K. Quarch, E. Durand, C. Schilde, A. Kwade and M. Kind, "Mechanical fragmentation of precipitated silica aggregates", Chemical Engineering Research and Design, 88, 12 (2010).

53. S. Musić, N. Filipović-Vinceković and L. Sekovanić, "Precipitation of amorphous SiO2 particles and their properties", Braz. J. Chem. Eng., 28, 1 (2011).

54. K.D. Dahm and D.J. Dahm, "Separating the Effects of Scatter and Absorption Using the Representative Layer", Journal of Near Infrared Spectroscopy, 21, 5 (2013).

55. R. Steponavičius and S.N. Thennadil, "Extraction of chemical information of suspensions using radiative transfer theory to remove multiple scattering effects: application to a model multicomponent system", Analytical chemistry, 83, 6 (2011).

56. E. Herremans, E. Bongaers, P. Estrade, E. Gondek, M. Hertog, E. Jakubczyk, N. Nguyen Do Trong, A. Rizzolo, W. Saeys, L. Spinelli, A. Torricelli, M. Vanoli, P. Verboven and B. Nicolaï, “Microstructure-texture relationships of aerated sugar gels: Novel measurement techniques for analysis and control", Innovative Food Science \& Emerging Technologies, 18 (2013).

57. R. XU, Particle characterization: light scattering methods. Kluwer academic publishers, Miami (2002). 

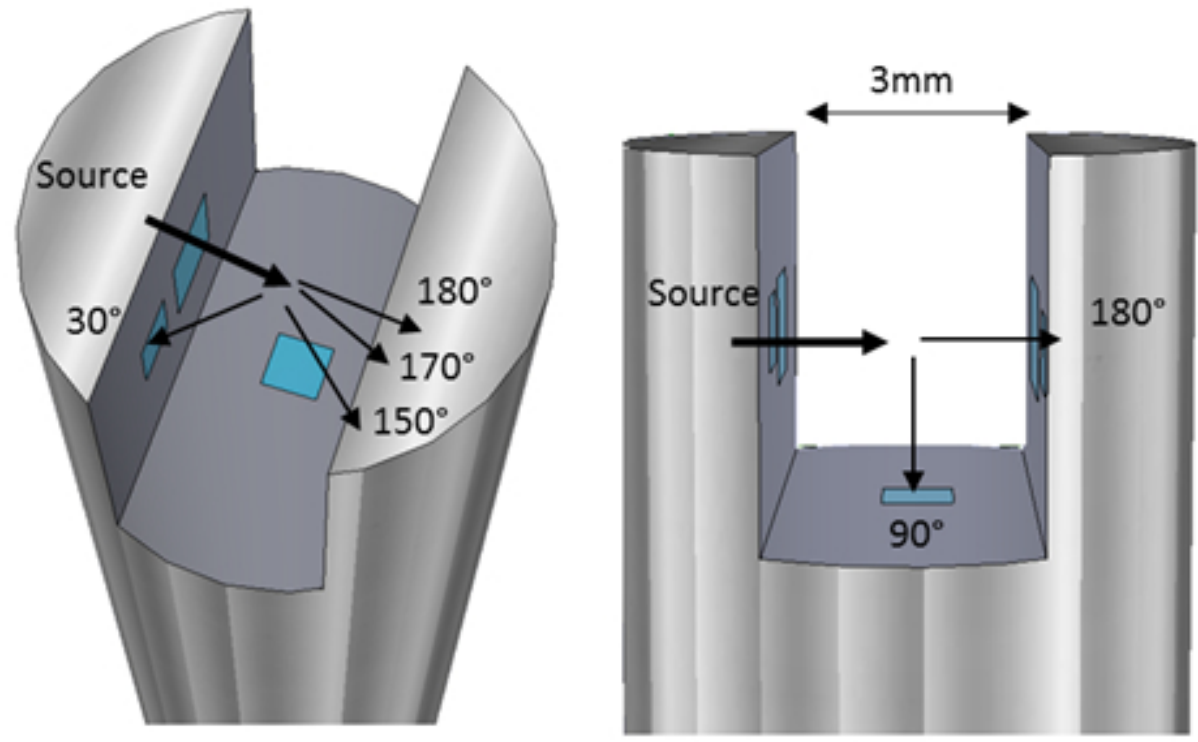

Multi-angle probe diagram (Sam-Flex, Indatech)

$81 \times 51 \mathrm{~mm}(150 \times 150 \mathrm{DPI})$ 


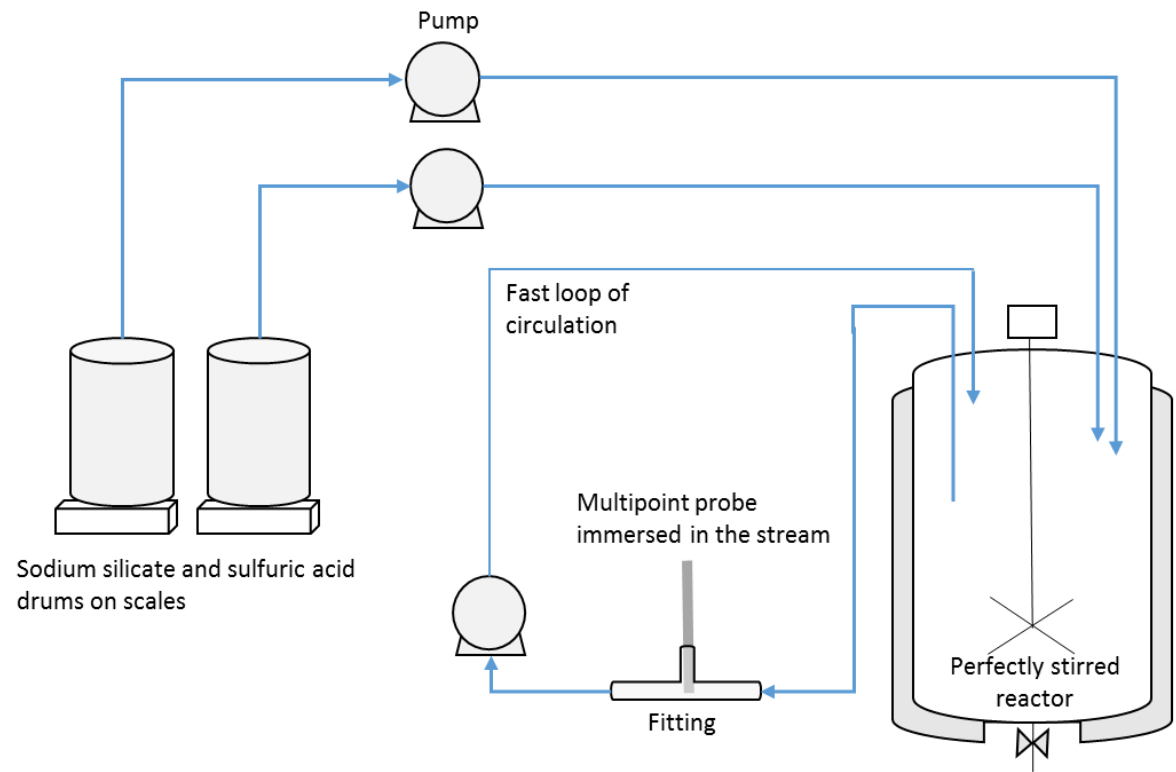

Experimental set up $192 \times 122 \mathrm{~mm}(150 \times 150 \mathrm{DPI})$ 


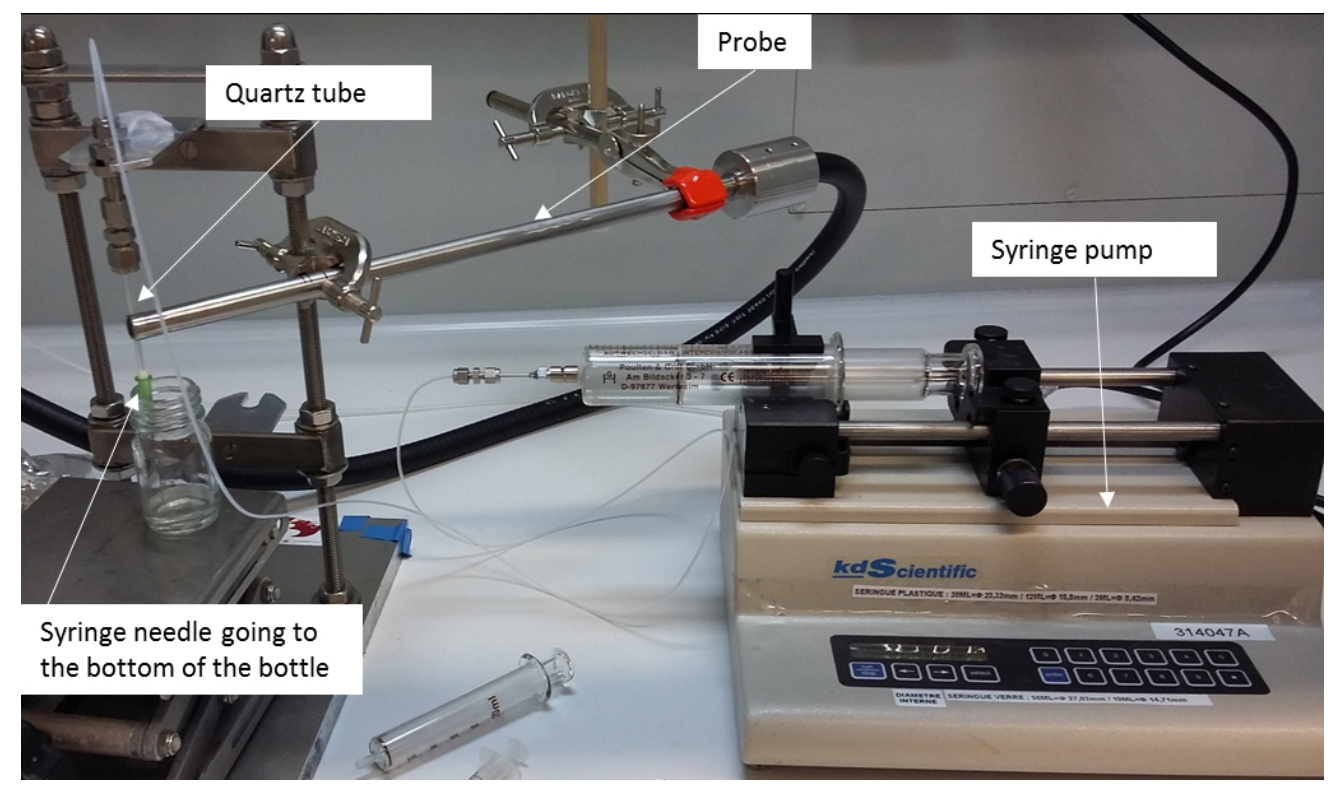

Photograph of the experimental set up

$173 \times 101 \mathrm{~mm}(150 \times 150 \mathrm{DPI})$ 

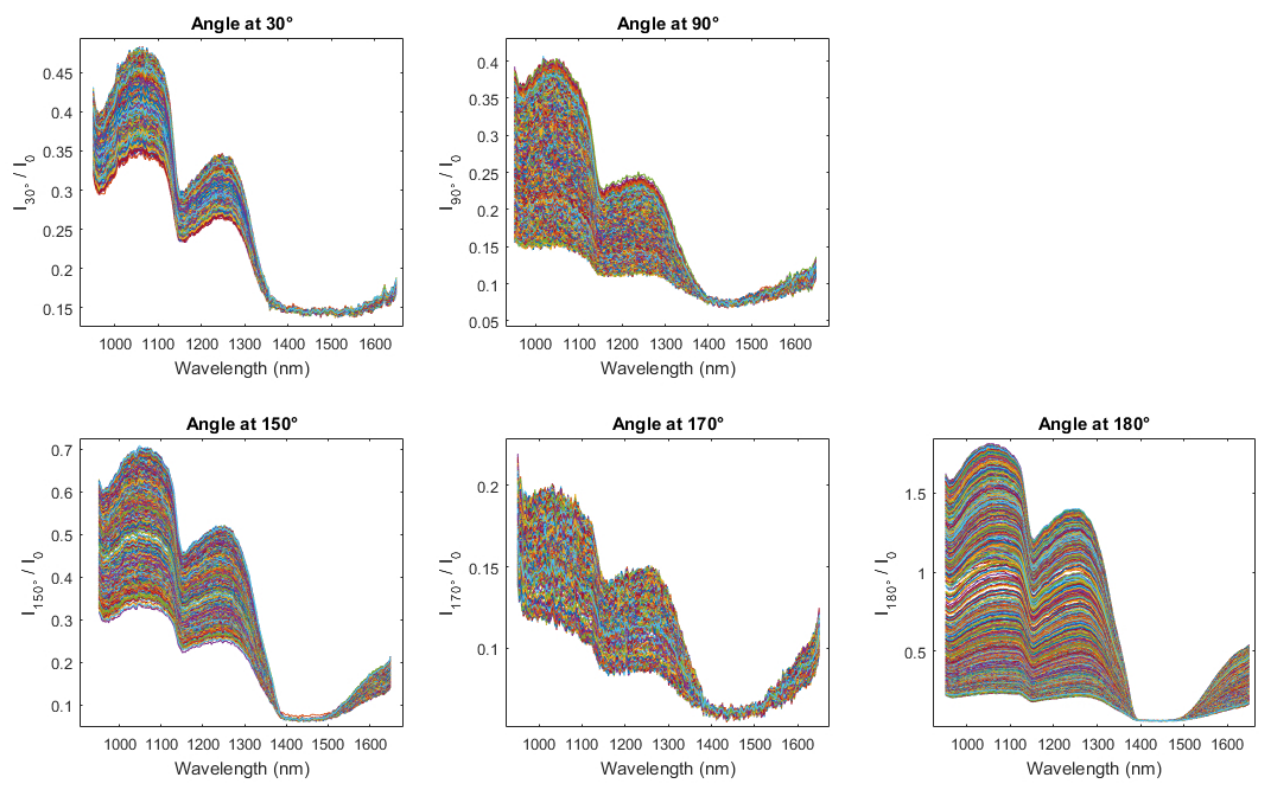

Spectra I/IO at the five angles throughout the batch $290 \times 182 \mathrm{~mm}(96 \times 96 \mathrm{DPI})$ 


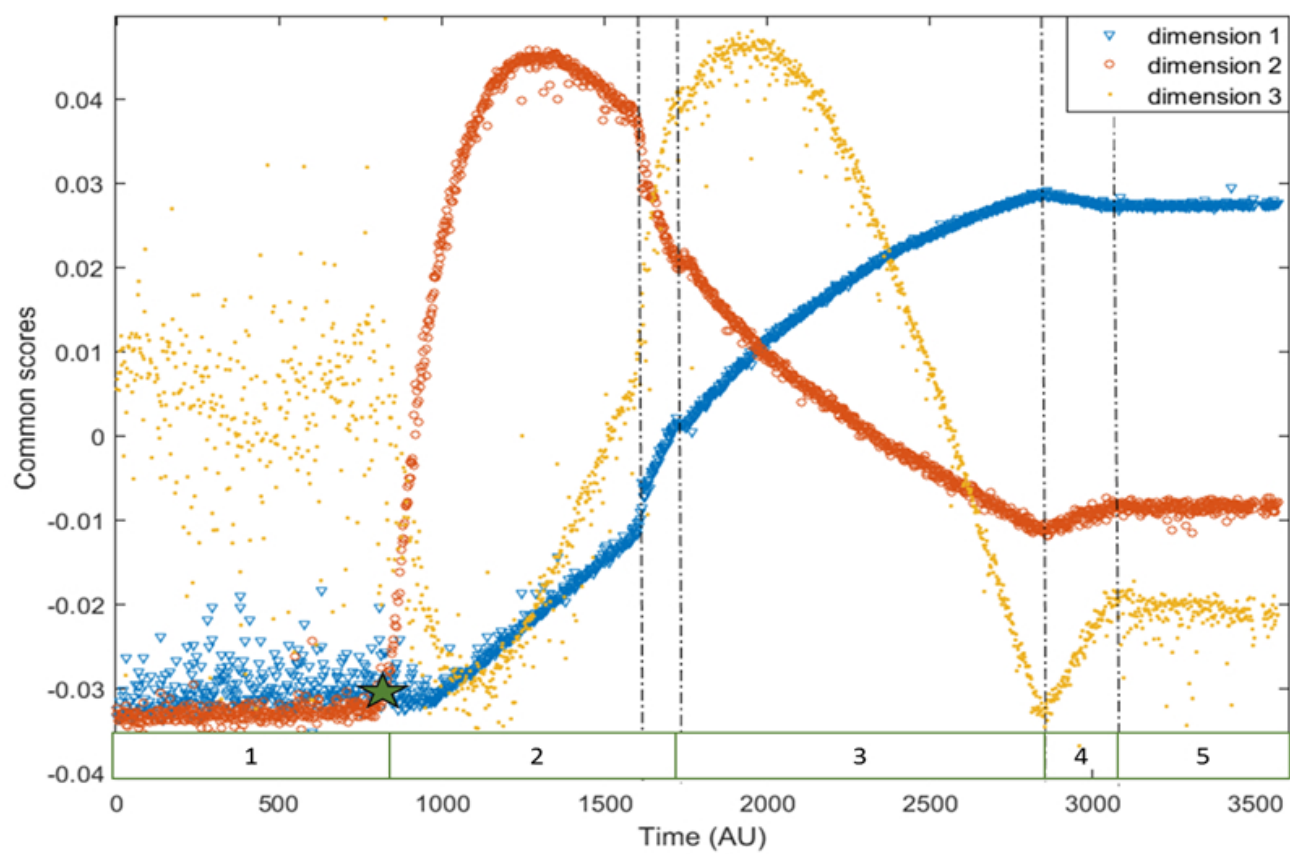

Common scores for three common dimensions according to time. The dotted lines correspond to the modifications of the process, the star and the numbered strips correspond to product evolutions

$135 \times 89 \mathrm{~mm}(150 \times 150 \mathrm{DPI})$ 


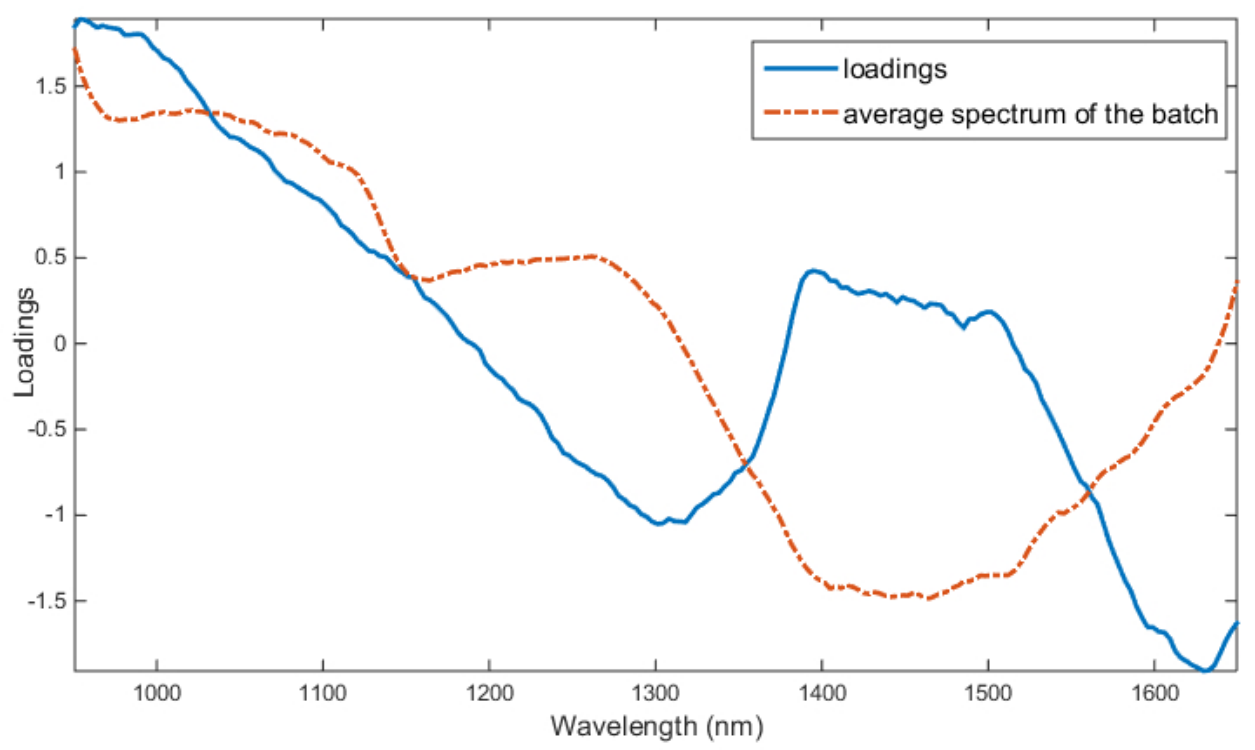

Average spectrum of the batch and loadings obtained for the angle $170^{\circ}$ in the second dimension $197 \times 143 \mathrm{~mm}(96 \times 96 \mathrm{DPI})$ 


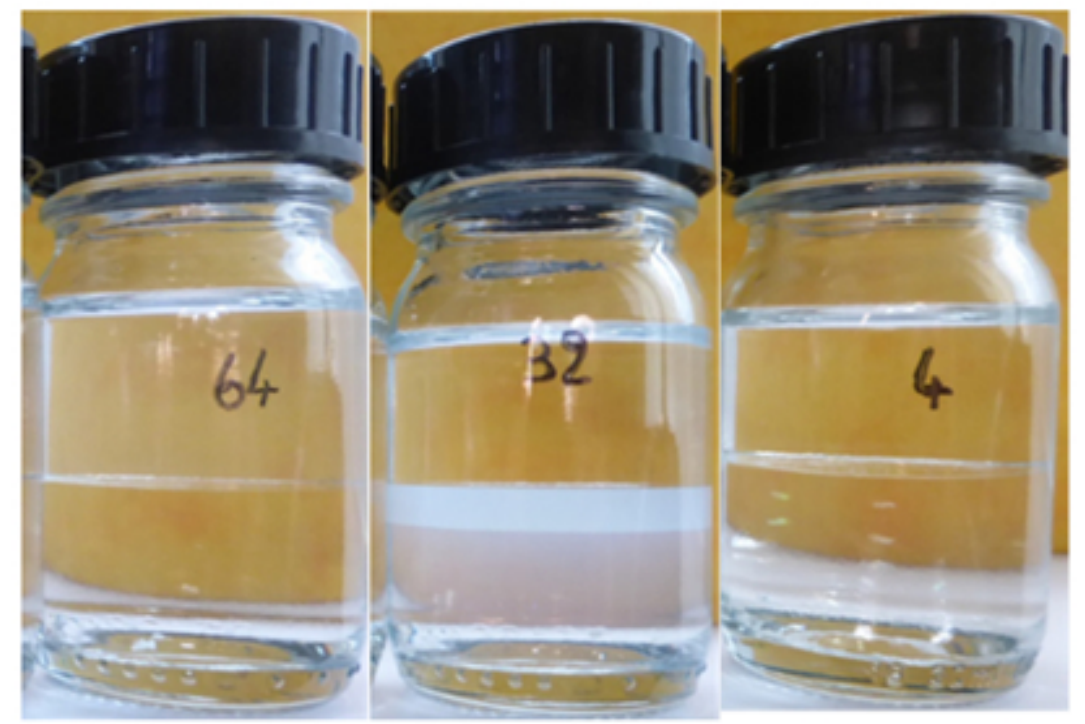

Photograph of the 3 samples for the micro-emulsion application

$74 \times 50 \mathrm{~mm}(150 \times 150 \mathrm{DPI})$ 


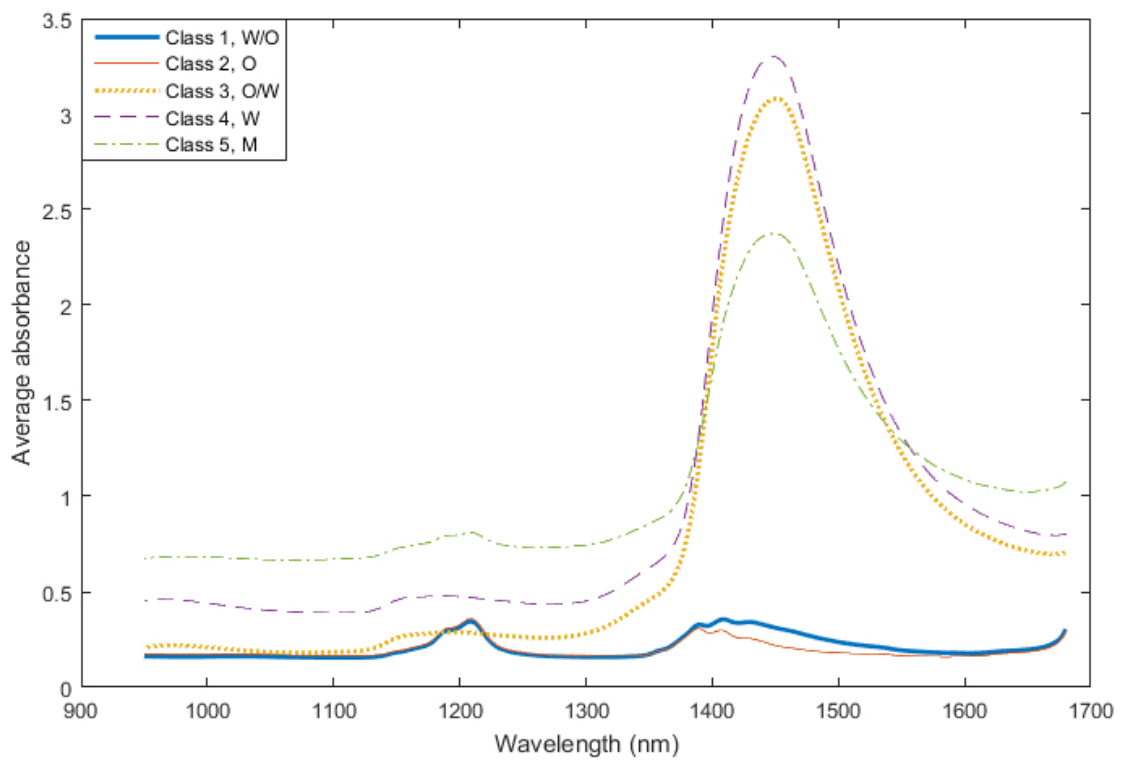

Mean absorption spectra of classes

$212 \times 134 \mathrm{~mm}(96 \times 96 \mathrm{DPI})$ 


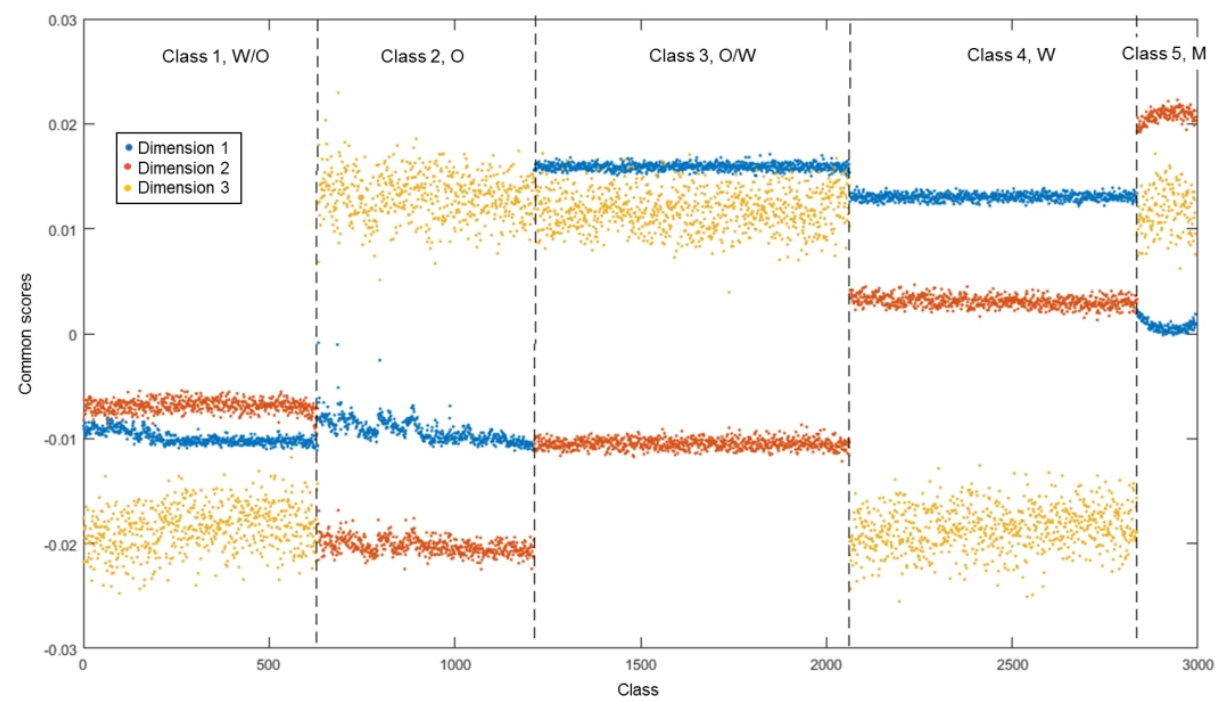

Common scores for three common dimensions according to the classes $306 \times 177 \mathrm{~mm}(150 \times 150$ DPI $)$ 

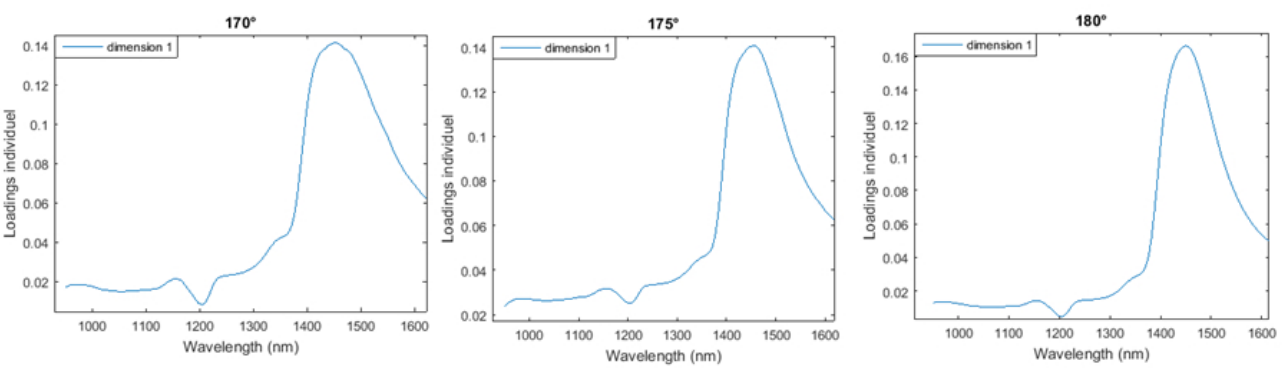

Individual loadings of transmission angles to the first dimension

$240 \times 70 \mathrm{~mm}(96 \times 96 \mathrm{DPI})$ 

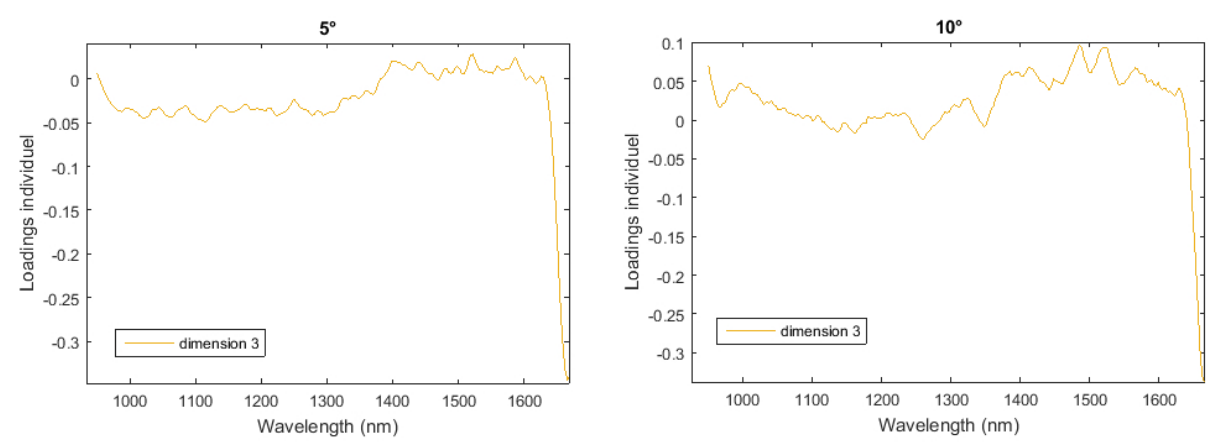

Individual loadings of angles at $5^{\circ}$ and $10^{\circ}$ of the third dimension

$278 \times 130 \mathrm{~mm}(96 \times 96 \mathrm{DPI})$ 